\title{
A Systematic Review and Meta-Analysis on Gualou Niubang Decoction for Acute Mastitis
}

Tian-tian Gao' ${ }^{1}$, Shuo Zhang ${ }^{2}$, Zhen-lin Chen ${ }^{*}$, Miao Wang ${ }^{3}$, Hong-yan Wang ${ }^{3}$, Wen-jing Ma ${ }^{1}$, Lu Wang ${ }^{1}$

${ }^{1}$ Shannxi University of Chinese Medicine, Xianyang 712046, Shannxi Province, China

2Beijing University of Chinese Medicine, Beijing 100029, China

${ }^{3}$ The Second Affiliated Hospital of Shannxi University of Chinese Medicine, Xianyang 712000, Shannxi Province, China

*Corresponding author: Zhen-lin Chen, chenzhenlin1997@163.com

Copyright: ( $) 2022$ Author(s). This is an open-access article distributed under the terms of the Creative Commons Attribution License (CC BY 4.0), permitting distribution and reproduction in any medium, provided the original work is cited.

\begin{abstract}
Objective: To systematically evaluate the clinical efficacy of Gualou Niubang decoction in the treatment of acute mastitis. Methods: Randomized controlled trials of Gualou Niubang decoction in the treatment of acute mastitis included in PubMed, Springer, Embase, Cochrane Library, CNKI, Wanfang, Weipu, and China Biomedical Literature Database from January 2016 to present. The Cochrane risk bias assessment tool was used to evaluate the risk of bias. Used Revman 5.3 software for Meta analysis. Results: There are 8 articles that meet the inclusion criteria, comprising 919 subjects, the test group were 462, cases and control group were 457 cases. The results of statistical analysis showed that the clinical efficacy of the experimental group was significantly higher than which of the control group, $[\mathrm{RR}=1.19,95 \% \mathrm{CI}(1.07,1.33), \mathrm{P}=$ 0.002], the difference was statistically significant. Among the secondary outcome indicators, compared to the control group, Gualou Niubang decoction can effectively relieve the main symptoms of acute mastitis $[\mathrm{MD}=-1.96,95 \% \mathrm{CI}(-2.27,-1.65)$, $\mathrm{P}<0.0001]$. Gualou Niubang decoction combined with antibiotics can shorten the length of hospital stay $[\mathrm{MD}=-5.23,95 \% \mathrm{CI}$ $(-6.02,-4.44), \mathrm{P}<0.00001]$ and reduce white blood cell count $[\mathrm{MD}=-2.40,95 \% \mathrm{CI}(-4.71,-0.08), \mathrm{P}=0.04]$ are better than antibiotics. Conclusion: The use of Gualou Niubang decoction to treat acute mastitis can not only improve clinical efficacy, but also effectively relieve clinical symptoms, shorten hospital stay and reduce white blood cell count.
\end{abstract}

Keywords: Gualou Niubang decoction; Acute mammary mastitis; Milk carbuncle; Meta analysis

Online publication: January 19, 2022

\section{Introduction}

Acute mastitis is a clinically common acute purulent infection of the breast. It is inflammation of the connective tissue in and around the mammary duct. It usually occurs in primiparous women, usually 3-4 weeks postpartum ${ }^{[1]}$, during and after 6 months of postpartum baby teething. It also often occurs in the time period of about a year ${ }^{[2]}$. Not only will it affect the quality of breastfeeding ${ }^{[3]}$, but it can also pose a serious threat to the health of mothers. The disease has a rapid onset and rapid progress. Failure to treat in time may result in sepsis or abscess formation and increase the suffering of the patient ${ }^{[4]}$. Western medicine mainly uses broad-spectrum antibacterial drugs and antipyretic and analgesic drugs as its conventional treatment options. Although the effect is acceptable, antibacterial drugs are not only prone to drug resistance, but also affect breastfeeding, resulting in low patient compliance. Acute mastitis belongs to the category of "mammary carbuncle" in Chinese medicine. A number of clinical studies have shown that Gualou Niubang decoction, as a classic prescription for the treatment of "mammary carbuncle," has good effects of soothing 
the liver, clearing the stomach, reducing swelling and clearing the milk. It is clinically effective in improving breast swelling and pain, breast lumps and breast local burning. Although there are some literature reports on the clinical efficacy of Gualou Niubang Decoction in the treatment of acute mastitis, these trials often have small sample sizes and single clinical trial results. Therefore, for the first time, this study adopted the method of literature collection and meta-analysis to screen out the literature related to acute mastitis in the existing clinical randomized controlled trials, and collected, sorted, and statistically analyzed the research results. A systematic review of the clinical efficacy of Gualou Niubang decoction in the treatment of acute mastitis provides a certain value of evidence-based reference for future clinical applications.

\section{Data and methods}

\subsection{Literature search}

The search period is from January 2016 to present. In Chinese, "Gualou Niubang decoction, breast carbuncle, externally blown breast carbuncle, breast blown, acute mastitis, lactation mastitis" is the keyword; Search in English with "acute mastitis, lactational mastitis, mastitis," "Gualou Niubang decoction, GualouNiubang, Traditional Chinese Medicine” as keywords. Search CNKI, Wanfang, Weipu, China Biomedical Literature Database, PubMed, Springer, EMbase, Cochrane Library. According to the characteristics of each database, the corresponding search strategy is adopted. The purpose of the search is to collect publicly published randomized controlled trials on the treatment of Gualou Niubang Decoction in the treatment of acute mastitis at home and abroad.

\subsection{Inclusion criteria}

(1) Clinical randomized controlled trials.

(2) The included patients meet the diagnostic criteria for acute mastitis.

(3) All patients are not limited to age and ethnicity.

(4) The treatment group is mainly Gualou Niubang Decoction.

(5) The control group was treated with conventional Western medicine or other treatments.

\subsection{Exclusion criteria}

(1) Repetitive articles with the same content are retrieved from different databases.

(2) Animal experiments or basic experiments.

(3) Sample data is unclear or incomplete.

\subsection{Data extraction}

Two researchers independently screened the titles and abstracts of potential eligible studies, and then they retrieved and reviewed the full text of the possible studies based on the inclusion and exclusion criteria and extracted the data. If there was disagreement, they agreed through discussion or submitted it to a third party for evaluation. Data extraction table mainly included the basic information of the study (author's name, title of the study, year of publication, country/ region, and publication status), study characteristics (sample size, source of cases, age, gender, distribution, diagnostic criteria, and inclusion and exclusion criteria), intervention and control measures (dosage form, dose, and duration),research methodology (random scheme generation, allocation hiding, blind method, incomplete result data, selective reporting, other biases, and loss of follow-up), and outcome measures.

\subsection{Assessment of literature quality}

Use Cochrane risk bias assessment tool to evaluate the risk of bias in the included literature ${ }^{[5]}$, two 
reviewers independently evaluated the methodological quality of each included study. It comprised the following seven aspects: random sequence generation, allocation concealment, blind method, incomplete result data, selective reporting, and other biases. The quality evaluation results of each item can be divided into three grades: "low risk" "high risk" and "unclear." Also, use the modified "Jadad scoring method." The methods for determining "low risk" are: randomized comparison table method, allocation concealment method, sufficiently reliable blinding method, complete result data, no selective report, no loss to followup and withdrawal.

\subsection{Statistical analysis}

Statistical analysis was performed using Rev Man 5.3 software ${ }^{[6]}$. The binomial variables were described by using relative risk (RR) and $95 \%$ confidence interval (CI), and the continuous variables were described by mean difference (MD) and 95\% CI to describe the effect value of the intergroup comparison. Heterogeneity was judged on the basis of the results of $\mathrm{I} 2$ test. $\mathrm{I}^{2}>50 \%$ indicated that the heterogeneity of inter-study was significant, and the random effect model was adopted. Additionally, the fixed effect model was adopted when $\mathrm{I}^{2}<50 \%$, it is believed that there is a large heterogeneity among the studies, and it is necessary to adopt a random effects model and analyze the sources of heterogeneity in the included studies. When $\mathrm{P}<0.05$, the result is considered to be statistically significant ${ }^{[7]}$.

\section{Results}

\subsection{Overview of included literature}

According to the search strategy, 147 original documents were retrieved in 7 databases, 104 articles remain after deleting duplicate documents,87 irrelevant articles are deleted after browsing the titles and abstracts. Initially obtained 17 documents that may meet the inclusion criteria, and finally included 8 randomized controlled trials after reading the full text ${ }^{[8-15]}$. The flow chart of literature retrieval and screening is shown in Figure 1.

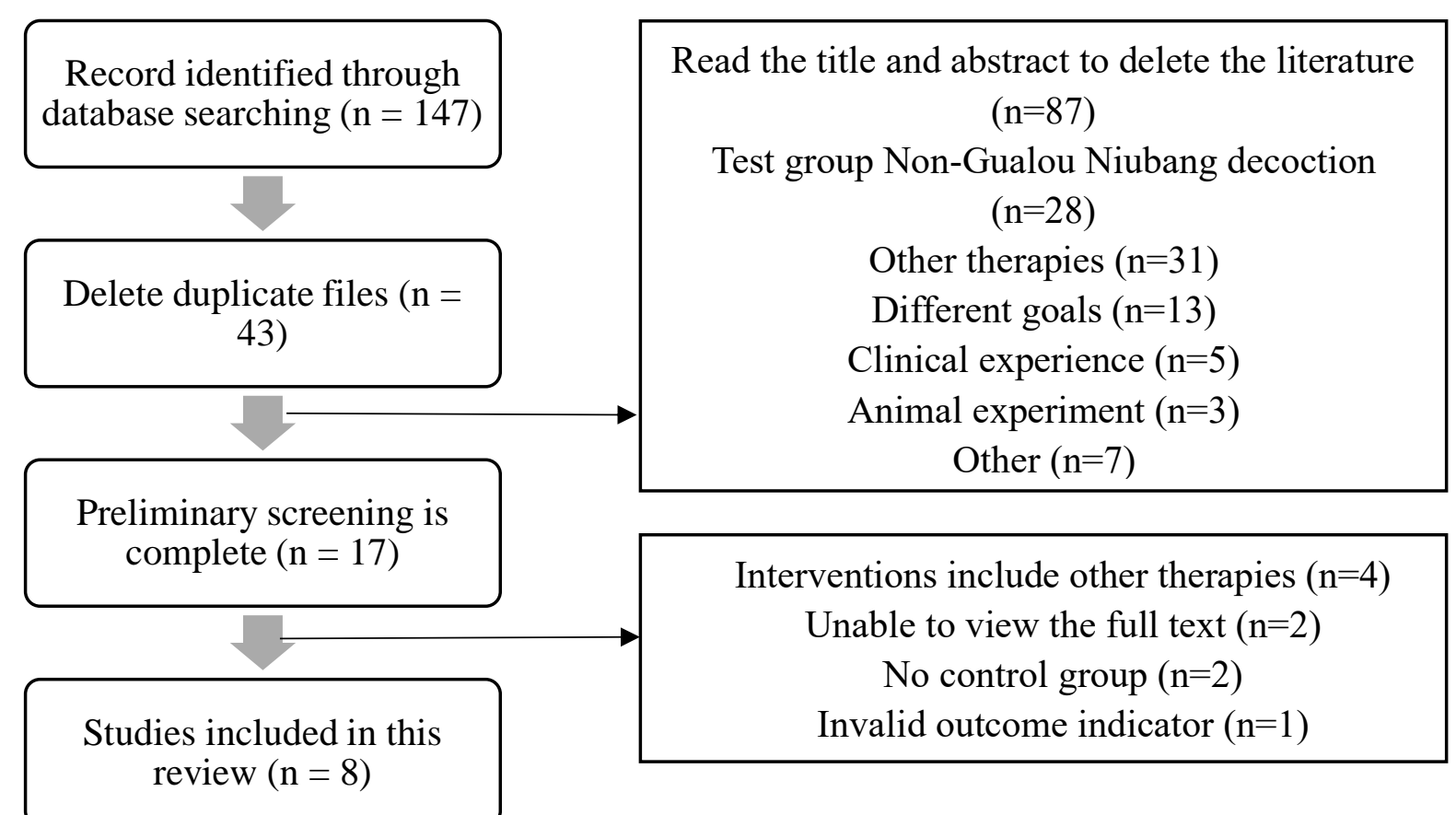

Figure 1. Flow diagram of literature selection 


\subsection{Basic characteristics of the included literature}

All trials included a total of 919 patients with acute mastitis. The sample size ranges from 60 to 200. In the treatment group, 3 trials ${ }^{[8-10]}$ only used Gualou Niubang decoction, 4 trials ${ }^{[11-14]}$ used this formula in combination with antibiotics. One trial ${ }^{[15]}$ used Gualou Niubang decoction combined with small needle knife to treat. The 8 included trials all described the clinical efficacy of Gualou Niubang decoction in the treatment of acute mastitis. Two trials ${ }^{[8,10]}$ mentioned relief scores for major clinical symptoms. Two trials ${ }^{[11,12]}$ involved changes in the length of hospital stay, and two other records ${ }^{[14,15]}$ recorded white blood cell counts before and after treatment.

\subsection{Results of quality evaluation and risk of bias assessment of included trials}

Evaluate the risk of bias in the included literature by using the standards in "Cochrane Intervention System Evaluation Manual." Only $2^{[13,15]}$ of the 8 documents stated that the random number table method was adopted, the rest of the literature only mentions random but does not specify the specific method of random sequence generation. Due to the general data (age, course of disease, time of onset, severity) of the test group and the control group were evaluated, the difference was not statistically significant $(\mathrm{P}>0.05)$, so judged as low risk. Also, 8 articles did not report on allocation concealment and blinding.

The number of reports before and after the 2 trials ${ }^{[11,14]}$ is inconsistent, determined as high risk. Use bias risk assessment tools to make a bias risk map for the included trials. Red represents high risk, yellow represents unclear, and green represents low risk. See Figure 2.
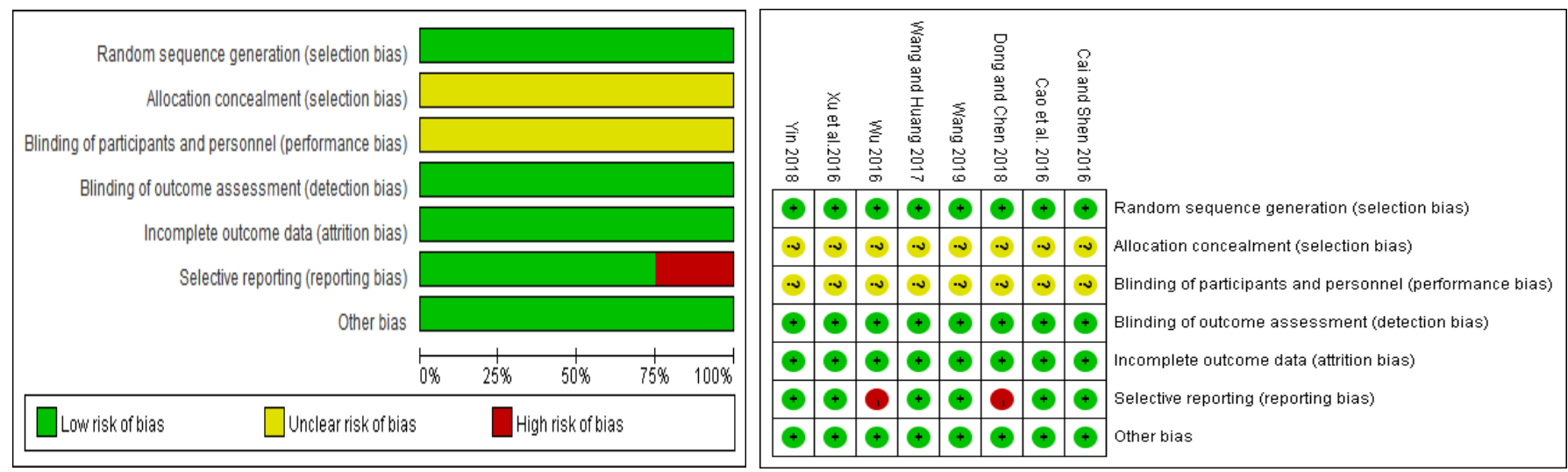

Figure 2. The results of the deviation risk evaluation of the included research literature

\subsection{Meta analysis results}

\subsubsection{Clinical efficacy analysis}

Except for $3^{[8,9,11]}$ of the 8 trials that used cured, effective, and ineffective, the effective criteria of other trials include healing, markedly effective, effective, and ineffective. Number of valid cases total number of cases-number of invalid cases. There was a total of 919 patients in 8 trials, test group: control group = 462:457, [RR=1.19,95\% CI $(1.07,1.33), \mathrm{P}=0.002]$. It shows that the clinical efficacy of Gualou Niubang decoction and its combination with antibiotics or combined with a small needle knife in the treatment of acute mastitis is significantly higher than that of using antibiotics or a small needle knife alone. The results are statistically significant. See Figure 3.

\subsubsection{Major symptom relief score}

There are 2 trials ${ }^{[10,13]}$ included in the literature to score the main symptoms according to "Modern Traditional Chinese Medicine Breast Diseases" and "Guiding Principles for Clinical Research of New 
Chinese Medicines." The main symptoms are breast tenderness, breast lumps, milk excretion, etc. Using a 4-level scoring method, 0 is scored as asymptomatic; mild, moderate, and severe symptoms are scored 1-3 points respectively. The lower the score, the more obvious the relief of symptoms. Use the major symptom relief score as an observation indicator, [MD $=-1.98,95 \% \mathrm{CI}(-2.27,-1.65), \mathrm{P}<0.00001]$, the main symptom relief score of the experimental group was lower than that of the control group, indicating that compared with the use of antibiotics, Gualou Niubangtang significantly alleviated the main clinical symptoms of the patients, and the difference was statistically significant. See Figure 4.

\subsubsection{Length of hospital stay}

Two trials ${ }^{[11,12]}$ compared the length of hospital stay between the experimental group and the control group, $[\mathrm{MD}=-5.23,95 \% \mathrm{CI}(-6.02,-4.44), \mathrm{P}<0.00001]$. The results show that compared with antibiotics, Gualou Niubang decoction can significantly shorten the length of hospital stay, and the difference is statistically significant. See Figure 5.

\subsubsection{White blood cell count}

There are also 2 trials ${ }^{[12,13]}$ that recorded the patient's white blood cell count before and after treatment, $[\mathrm{MD}=-2.40,95 \% \mathrm{CI}(-4.71,-0.08), \mathrm{P}=0.04]$. This shows that compared with the control group, Gualou Niubangtang has more advantages in reducing white blood cell count, and the outcome is statistically significant. See Figure 6.

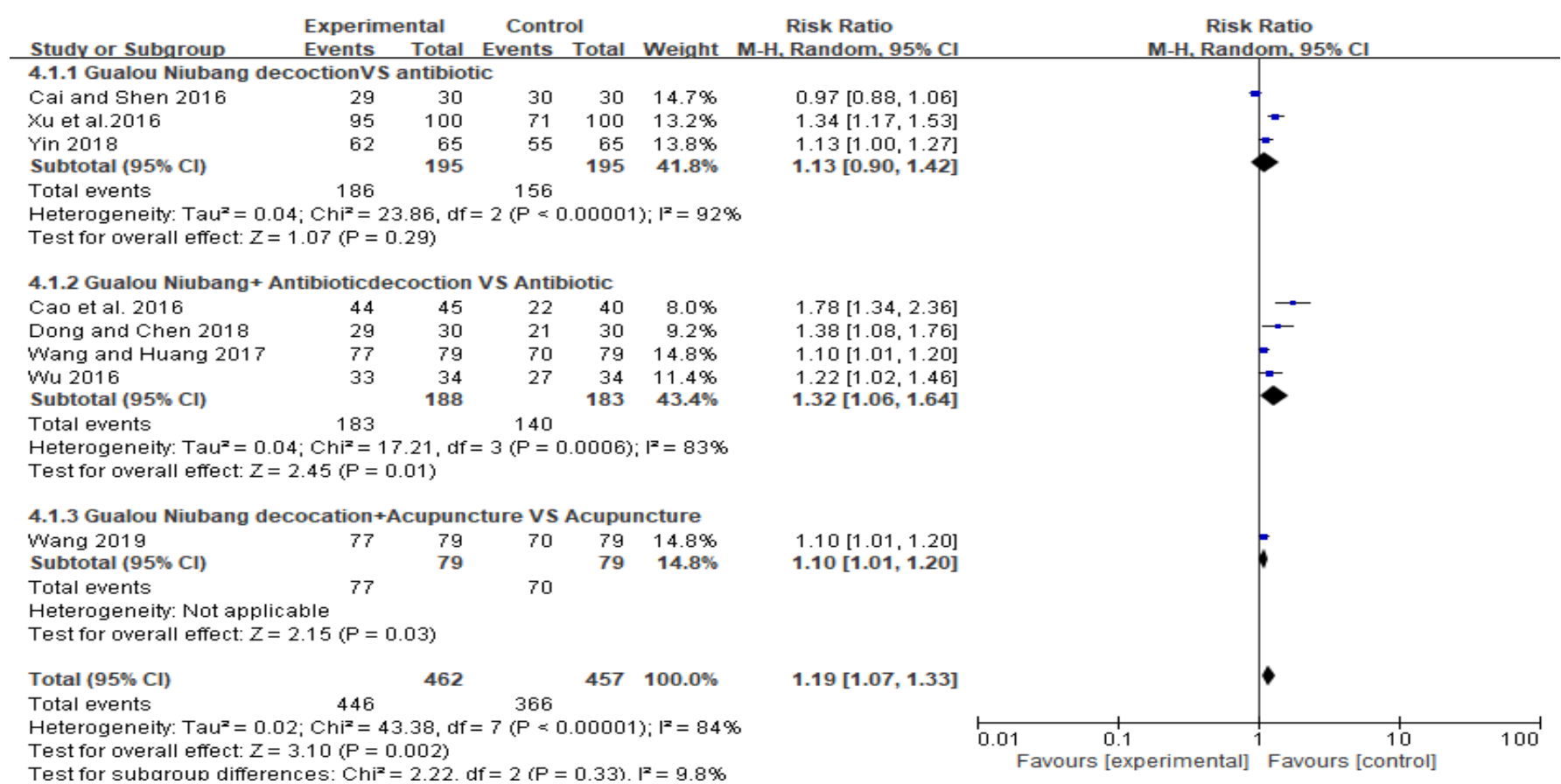

Figure 3. Clinical effect forest diagram

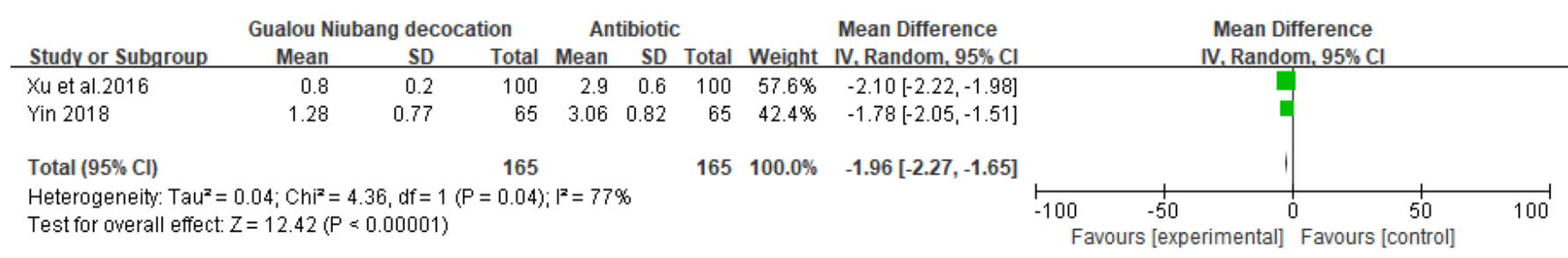

Figure 4. Forest diagram of major symptom relief scores 


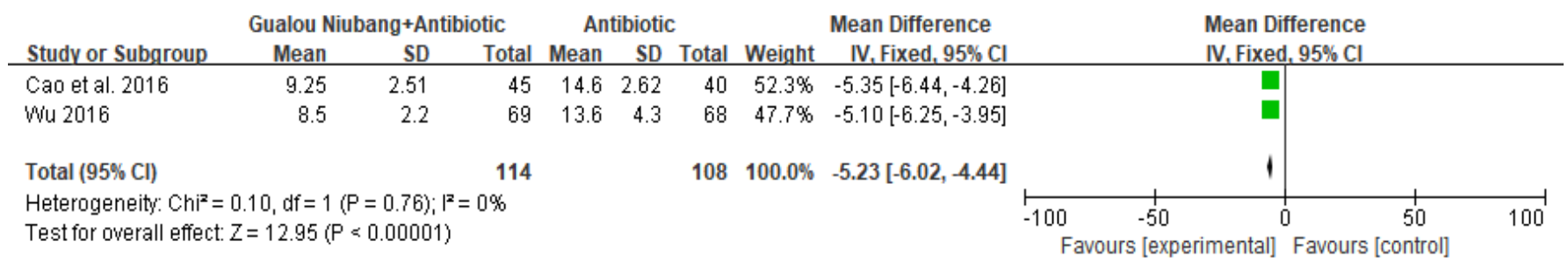

Figure 5. Forest diagram of length of hospital stay

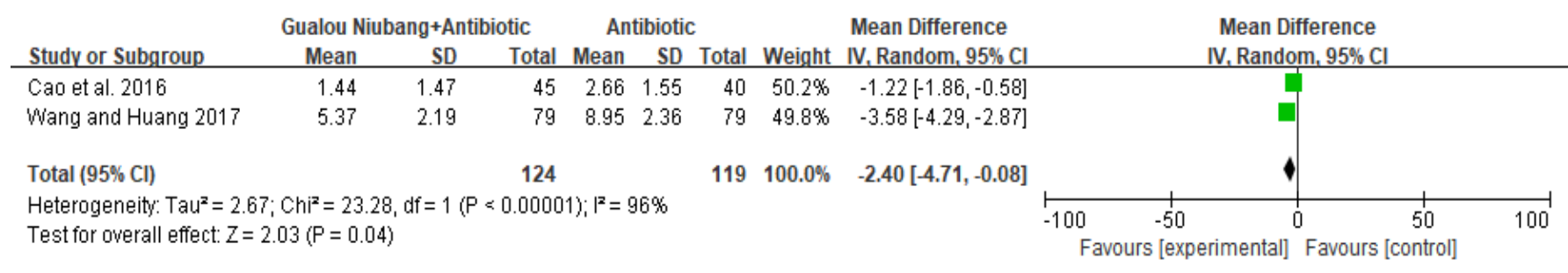

Figure 6. White blood cell count forest plot

\section{Discussion}

Acute mastitis is a bacterial infection caused by milk stasis and bacterial invasion. It is a common disease during lactation ${ }^{[16]}$. This disease will not only bring great pain to the parturient, but also bring hidden dangers to the safety of breastfeeding, which is extremely detrimental to the mother and the baby ${ }^{[17]}$. Western medicine advocates the use of sufficient antibiotics as soon as possible to reduce inflammation and control infections and prevent the further development of abscesses ${ }^{[18]}$. However, related research believes that, early use of a large number of antibiotics can easily cause local qi and blood coagulation, and the organization of inflammatory tissues, form a "stiff block." At the same time, most patients are concerned about the safety of infant breastfeeding and are reluctant to use antibiotics ${ }^{[19]}$. Therefore, a treatment with definite curative effect, less side effects, more acceptable to patients, and alleviating the adverse reactions caused by antibiotics can benefit many patients with acute mastitis.

Chinese medicine believes that acute mastitis belongs to the category of "Milk Carbuncle." The etiology and pathogenesis of this disease are mostly postpartum emotional depression, stagnation of liver qi, stagnation of heat in the stomach of Yangming, block of milk collaterals, and milk congestion caused by difficulty in expelling milk ${ }^{[20]}$. Gualou Niubang Decoction is a classic prescription in "Yi Zong Jin Jian." Gualou and Niubangzi seeds are both entered into the stomach meridian. Among them, Gualou disperses knots and broad breasts, and the bitter cold of burdock seeds can clear away heat, relieve fire and detoxify. The two are the master medicine, which has the effect of removing evil and relieving depression ${ }^{[21]}$. Bupleurum has the powers of reconciling the exterior and interior, soothing the liver and promoting yang. Green peel and tangerine peel can regulate qi, invigorate the spleen, relieve depression and eliminate heat; honeysuckle and forsythia have good effects on clearing away heat, detoxification, swelling and dispelling lumps, especially for breast carbuncle. Significantly ${ }^{[22]}$, trichosanthes clears away heat and relieves carbuncle and dispels carbuncle, scutellaria and gardenia clear heat and detoxify, ignites downwards, saponins thorn dispels carbuncle and dispels carbuncle, and licorice reconciles various medicines. Combination of multiple drugs to achieve the effects of dredging the collaterals and lowering the breast, clearing away heat, reducing swelling and dispelling congestion ${ }^{[23]}$.

The eight studies in this study all use clinical efficacy as their main outcome indicator. The study shows that the experimental group is significantly better than the control group in terms of clinical efficacy. Also, in further research under the subgroup, it was found that. Therefore, in the future clinical work, the treatment of acute mastitis can be combined with the patient's condition and the Gualou Niubang decoction can be used to effectively relieve the breast pain of the mother and improve the confidence of the mother to 
breastfeed, conducive to the outcome of the disease, beneficial to both mothers and newborns. The shortcomings of this study are: the sample size reported in the existing literature is small, and randomized controlled trials do not accurately report allocation concealment, blinding, etc., and there is a certain degree of bias. Therefore, when selecting documents, read carefully and strictly screen them in order to obtain more complete data. It is hoped that future studies can include larger, more rigorous and complete randomized controlled trials, reduce the influence of other factors, and provide a more reliable basis for clinical practice.

\section{Funding}

Fund Project: General Project of the National Natural Science Foundation of China (No. 81974584)

\section{Disclosure statement}

The author declares no conflict of interest.

\section{References}

[1] Zhu XQ, Mi HX, Lin X, et al., 2017, Effect of Gualou Niubang Decoction Combined with Yanghe Decoction Combined with External Application of Traditional Chinese Medicine on the Levels of IL6 and CRP in Early Lactation Patients with Acute Mastitis. Chinese Archives of Tradtional Chinese Medicine, 35(4): 819-821.

[2] Peng FH, Yun HX, 2014, Clinical Observation on 43 Cases of Acute Mastitis Treated by Oral and External Application of Traditional Chinese Medicine. Herald of Traditional Chinese Medicine, 20(14): 70-71.

[3] Guo HY, Wang EL, Zhong CE, 2015, Effect Observation and Nursing of Self-Made Chinese Medicine External Application in Adjuvant Treatment of Lactation Acute Mastitis. Nursing Practice and Research, 12(5): 145-146.

[4] Liu S, Zhao HM, Gao Y, 2019, The Effect of the Combined Application of Gualou Niuang Decoction and Yanghe Decoction with External Application of Traditional Chinese Medicine on the Levels of Inflammatory Mediators and TCM Syndrome Scores in Patients with Acute Mastitis During Lactation. China Pharmacoeconomics, 14(11): 110-112+116

[5] Qi J, Zhang YF, Xia QQ, 2015, Erchen Decoction in the Treatment of Nonalcoholic Fatty Liver Disease: A Systematic Review. Liaoning Journal of Traditional Chinese Medicine, 31(212): 2276-2280.

[6] Higgins JP, Thompson SG, 2002, Quantifying Heterogeneity in a Meta-Analysis. Stat Med, 21(11): 1539-1558.

[7] Higgins JPT, Ompson SG, et al., 2003, "Measuring Inconsistency in Meta-Analysis," BMJ, 327(7414): $557-560,2003$.

[8] Yin LX, 2018, Clinical Effect Analysis of Modified Gualou Niubang Decoction Combined with Milk Excretion in the Treatment of Lactation Mastitis. China's Rural Health, (15): 68+67.

[9] Cai GY, Yang LC, 2016, Clinical Observation of Effects of Modified Gualou Niubang Decoction on Early-Stage Acute Mastitis During Lactation. Shanghai Journal of Traditional Chinese Medicine, 50(12): 57-59.

[10] Xu XQ, et al., 2016, Clinical Research of Modified Gualou Niubang Decoction Combined with Milk Ejection Method in Treating Lactation Mastitis. Clinical Journal of Chinese Medicine, 8(03): 73-75.

[11] Wu XB, 2016, Clinical Observation of Gualou Niubang Decoction in the Treatment of Acute Mastitis. Chinese Medicine Modern Distance Education of China, 2016. 14(12): 92-93. 
[12] Cao YN, Fan W, Wang H, 2016, Gualou Niubang Decoction Combined with Western Medicine in the Treatment of Early Acute Mastitis Analysis of the Curative Effect and Prognosis of the Patients. Journal of Emergency in Traditional Chinese Medicine, 25(11): 2158-2160.

[13] Wang RH, Huang, 2017, Clinical Effect Observacation of Gualou Niubang Decocation in the Treatment of Acute Mastitis in Lavtation Period. Shaanxi Journal of Traditional Chinese Medicine, 38(07): 823-824.

[14] Dong WQ, Chen DJ, 2018, Effect of Modified Gualou Niubang Decoction in the Treatment of Lactation Mastitis. Shenzhen Journal of Integrated Traditional Chinese and Western Medicine, 28(24): 32-34.

[15] Wang XJ, 2019, Clinical Study on the Treatment of Acute Suppurative Mastitis with Gualou Niubang Decoction Combined with Small Needle Knife Therapy. Henan Traditional Chinese Medicine, 39(06): 910-913.

[16] Lv JQ, Yang JJ, Gong XN, et al., 2014, Clinical Observation of Acute Suppurative Mastitis Complicated with Severe Infection During Pregnancy. Maternal and Child Health Care of China, 29(015): 2404-2405.

[17] Xia HL, Yang LC, 2011, Risk Factors of Puerperal Mastitis. Anhui Medical and Pharmaceutical, 15(12): $1522-1524$.

[18] Harris, 2006, Mammology USA, Shandong Science and Technology Press, Jinan, 3: 51.

[19] Zhou M, Lin X, Cheng YQ, et al., 2009, A Randomized Controlled Clinical Study on the Treatment of Acute Mastitis by Kneading Powder. Journal of Integrated Chinese and Western medicine, 7(12): 11301133.

[20] Gu BH, 1994, Practical Surgery of Traditional Chinese Medicine, Shanghai Scientific and Technical Publishers, Shanghai, 129.

[21] Meng JC, 2019, Clinical Observation on the Treatment of Mastitis (Stagnation) in Lactation with Gualou Niubang Decoction and Massage. Guangming Traditional Chinese Medicine, 34(15): 23562357.

[22] Jing YS, Wan H, 2016, Treatment of Acne Mastitis by Clearing Phlegm Dampness Combined with External Therapy. Jilin Traditional Chinese Medicine, 36(6): 583-585.

[23] Guo CM, Fu MG, Li D, et al., 2015, Clinical Analysis of 138 Cases of Non-Lactation Mastitis. Medical Review, 21(6): 1106-1107. 